\title{
Controlling bias in genomic breeding values for young genotyped bulls
}

\author{
S. Tsuruta, ${ }^{1 *}$ D. A. L. Lourenco, ${ }^{1}$ Y. Masuda, ${ }^{1}$ I. Misztal,,${ }^{1}$ and T. J. Lawlor ${ }^{2}$ \\ ${ }^{1}$ Animal and Dairy Science Department, University of Georgia, Athens 30602 \\ ${ }^{2}$ Holstein Association USA Inc., Brattleboro, VT 05301
}

\section{ABSTRACT}

The objectives of this study were to investigate bias in genomic predictions for dairy cattle and to find a practical approach to reduce the bias. The simulated data included phenotypes, pedigrees, and genotypes, mimicking a dairy cattle population (i.e., cows with phenotypes and bulls with no phenotypes) and assuming selection by breeding values or no selection. With the simulated data, genomic estimated breeding values (GEBV) were calculated with a single-step genomic BLUP and compared with true breeding values. Phenotypes and genotypes were simulated in 10 generations and in the last 4 generations, respectively. Phenotypes in the last generation were removed to predict breeding values for those individuals using only genomic and pedigree information. Complete pedigrees and incomplete pedigrees with $50 \%$ missing dams were created to construct the pedigree-based relationship matrix with and without inbreeding. With missing dams, unknown parent groups (UPG) were assigned in relationship matrices. Regression coefficients $\left(b_{1}\right)$ and coefficients of determination $\left(R^{2}\right)$ of true breeding values on $(G) E B V$ were calculated to investigate inflation and accuracy in GEBV for genotyped animals, respectively. In addition to the simulation study, 18 linear type traits of US Holsteins were examined. For the 18 type traits, $b_{1}$ and $R^{2}$ of GEBV with full data sets on GEBV with partial data sets for young genotyped bulls were calculated. The results from the simulation study indicated inflation in GEBV for genotyped males that were evaluated with only pedigree and genomic information under BLUP selection. However, when UPG for only pedigree-based relationships were included, the inflation was reduced, accuracy was highest, and genetic trends had no bias. For the linear type traits, when UPG for only pedigreebased relationships were included, the results were generally in agreement with those from the simulation study, implying less bias in genetic trends. However, when including no UPG, UPG in pedigree-based rela-

Received April 11, 2019.

Accepted July 16, 2019.

*Corresponding author: shogo@uga.edu tionships, or UPG in genomic relationships, inflation and accuracy in GEBV were similar. The results from the simulation and type traits suggest that UPG must be defined accurately to be estimable and inbreeding should be included in pedigree-based relationships. In dairy cattle, known pedigree information with inbreeding and estimable UPG plays an important role in improving compatibility between pedigree-based and genomic relationship matrices, resulting in more reliable genomic predictions.

Key words: genomic prediction, unknown parent group, US Holstein

\section{INTRODUCTION}

When predicting genomic estimated breeding values (GEBV) with a single-step algorithm, phenotypes, genotypes, and pedigrees can be included in one mixed model. However, inflation in genomic predictions for young genotyped bulls in dairy cattle has been reported when those bulls have no daughters with phenotypes (Aguilar et al., 2010; Tsuruta et al., 2011; Masuda et al., 2018). One reason for the bias could be preselection of those young genotyped bulls (Vitezica et al., 2011). Another could be incompatibility between the pedigree-based relationship matrix $(\mathbf{A})$ and the genomic relationship matrix $(\mathbf{G})$. When utilizing all available information in the single-step genomic (ssG) BLUP, $\mathbf{A}$ and $\mathbf{G}$ are constructed using all available animals in pedigree and only genotyped animals in the current population, respectively. Definitions of $\mathbf{A}$ and $\mathbf{G}$ also differ (i.e., expected relationships in $\mathbf{A}$ and realized relationships in $\mathbf{G}$ ), creating disparate distributions. As a result, inbreeding coefficients in $\mathbf{A}$ and $\mathbf{G}$ are often significantly different. Moreover, genotyped animals may have unknown parent(s) in $\mathbf{A}$ but not in G by definition of identity by state. When unknown parent groups (UPG) are included in the model, they need to be estimated accurately with sufficient information. The incompatibility between $\mathbf{A}$ and $\mathbf{G}$ and ill-defined UPG can cause biases in GEBV. To reduce the bias in GEBV, Koivula et al. (2018) excluded genotyped bulls with no progeny from the evaluation. Without progeny, those bulls have no major contribu- 
tions to other animals. One of the main purposes of progeny testing for dairy bulls in the genomic era is to evaluate young genotyped bulls as early as possible to shorten the generation interval significantly, as well as to increase accuracy in GEBV by using genomic information. Therefore, these young genotyped bulls need to remain in progeny testing for (re)evaluation. In addition, several heifers have been recently genotyped. These bulls and heifers have to be included in genomic evaluation at least until the bulls have no semen and the heifers are culled.

The objectives of this study were to investigate possible causes of bias in genomic predictions for dairy cattle, especially young genotyped bulls with no progeny and to find a practical approach to predict GEBV with less bias. A simulation study was conducted mimicking a dairy cattle population under varying assumptions for selection, pedigree structure, and distributions of phenotypes and genotypes. True breeding values were used for validation of genomic predictions in the simulation. Genomic predictions for linear type traits in US Holsteins were also conducted using different definitions of UPG, including inbreeding coefficients in $\mathbf{A}$ with and without considering unknown parents. The results from the US Holstein population were compared with those in genomic predictions from the simulation study.

\section{MATERIALS AND METHODS}

\section{Simulation}

Phenotypes, genotypes, and pedigrees were simulated with QMSim (Sargolzaei and Schenkel, 2009), mimicking a dairy cattle population where only females have phenotypes. Creating a historical population in 100 generations, 100 males and 50,000 females were assumed to be founders (Figure 1). In the next 10 generations, $10 \%$ of males and $50 \%$ of females were mated to produce 50,000 offspring in each generation. The heritability was assumed to be 0.5 , where half of the heritability was attributed to 580 QTL (20 QTL/chromosome). Each QTL effect was randomly generated based on half the additive genetic variance $\left(\sigma_{a}^{2}=50\right)$ assuming a gamma distribution with the shape parameter $=0.4$ (Hayes and Goddard, 2001). The other half was assumed to be polygenic inheritance that was not attributed to these 580 QTL. Across 29 autosomes, 58,000 SNP markers were generated. All chromosomes had the same genome length (2,000 SNP per chromosome). In the first historical population, all allele frequencies were equal. The informativeness of each SNP marker in each generation can change due to mutation, genetic drift, and selection. The simulated data sets in 2 scenarios (a) and (b) were described in Figure 1 as follows: (a) The number of generated animals was 50,000 per generation for males and females, and the top $10 \%$ and $50 \%$ in EBV were selected for males and females, respectively, to produce offspring in the next generation. The number of animals in pedigree was 400,640 including generation 0 (founders); the total number of phenotypes was 225,000 in generations 1 to 9 after removing phenotypes in the last generation 10. All $(200,000)$ animals in the last 4 generations from 7 to 10 were genotyped.

(b) The same population structure as in scenario (a) with no artificial selection.

In the 2 scenarios, (G)EBV were calculated with no missing parents or with $50 \%$ missing dams that were randomly selected. With missing dams, UPG were assigned based on generations of missing dams, and results from 2 models with and without UPG were compared. Original inbreeding coefficients without considering unknown parent(s) (2.0 and $0.6 \%$ on average across all 10 generations and all 10 replicates for (a) and (b), respectively) and approximated inbreeding coefficients with considering unknown parents [3.4 and $0.5 \%$ on average for (a) and (b), respectively] were included in $\mathbf{A}$. The original inbreeding coefficient was calculated based on relationships including unknown pedigrees, whereas the approximated inbreeding coefficient was calculated using relationships among UPG according to Aguilar and Misztal (2008). As a result, the approximated inbreeding could be higher than the original inbreeding. Both scenarios were replicated 10 times. For validation of genomic predictions, regression coefficients $\left(\mathbf{b}_{\mathbf{1}}\right)$ and coefficients of determination $\left(\mathbf{R}^{2}\right)$ were calculated from true breeding values $(\mathbf{T B V})=\mathrm{b}_{0}$ $+b_{1} \times(G) E B V$.

\section{Linear Type Traits in US Holsteins}

Eighteen linear type traits in US Holsteins were used to investigate bias and accuracy in GEBV for young genotyped bulls with no daughters in 2010. The data for the national genetic evaluation in August 2014 were provided by Holstein Association USA Inc. (Brattleboro, VT). For genomic evaluation, 10,067,745 phenotypic records for 6,550,442 cows including 9,730,943 animals in pedigree and 58,990 SNP markers for 569,404 genotyped animals were used. The genomic data were provided by USDA-Agricultural Research Service (Beltsville, MD) in 2014 before the Council on Dairy Cattle Breeding (Bowie, MD) started managing all genotypes for US dairy cattle. The animal model used in national evaluation for type traits in US Holsteins is described in Tsuruta et al. (2001). Unknown parent 


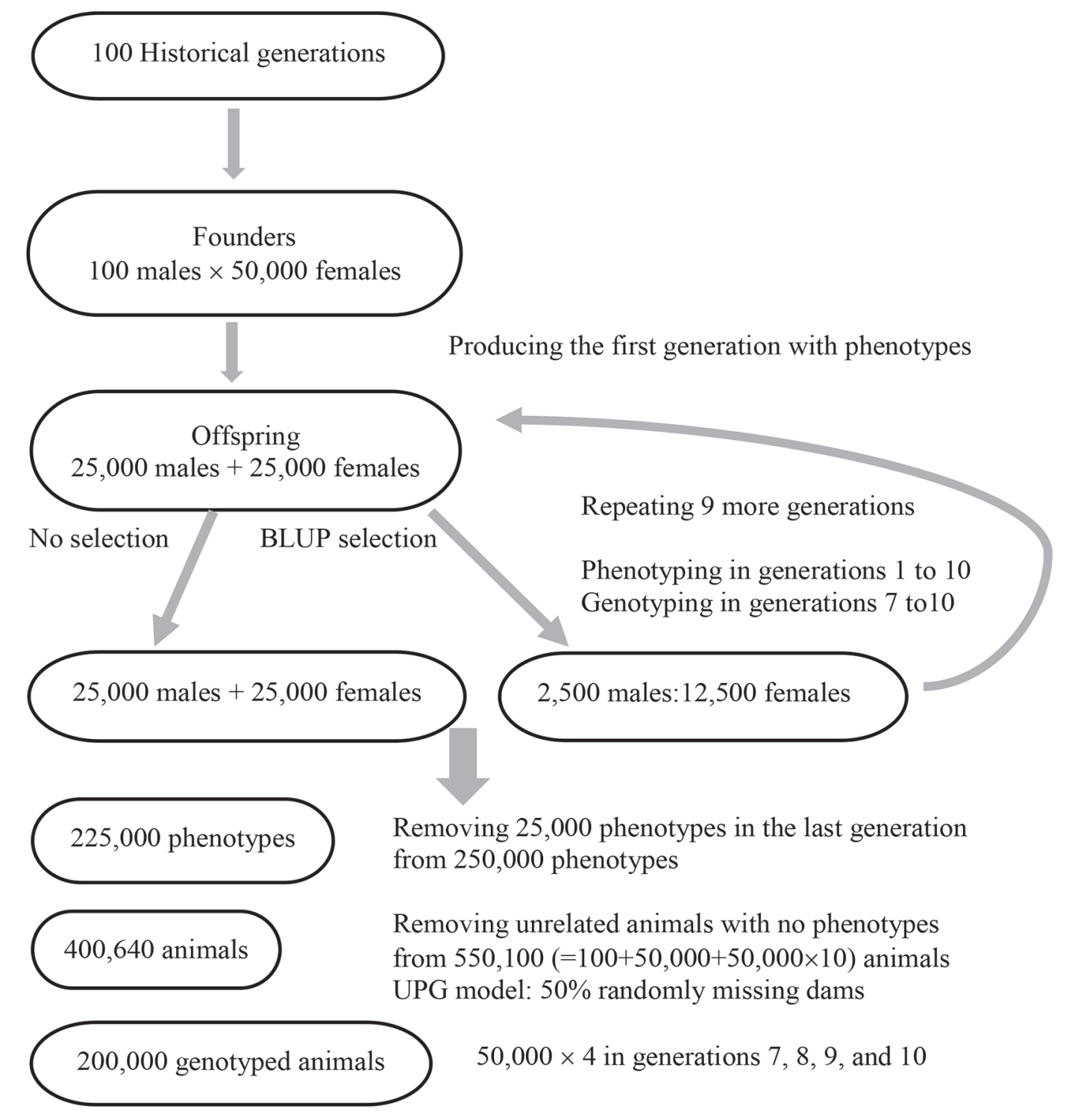

Figure 1. Diagram of simulation scenarios. UPG = unknown parent group.

groups in type traits based on sex (sire and dam) and birth-year group (every $4 \mathrm{yr}$ for sire and every $2 \mathrm{yr}$ for dam) were defined as in Table 1. Unknown sires and dams represented 10 and $28 \%$ of all animals in the pedigree, respectively. The original inbreeding coefficient was $2.6 \%$ on average, whereas the approximated inbreeding coefficient assuming relationships among unknown parents was 5.5\%. Genomic predictions for 18 type traits were calculated using phenotypes up to 2010 with all genotypes and pedigree up to 2014, and the full phenotypic data up to 2014 were used for validation. As indicators of inflation (or deflation) and accuracy in GEBV,$b_{1}$ and $R^{2}$ from DYD $=b_{0}+b_{1} \times$ GEBV were obtained, respectively, where DYD were daughter yield deviations (Mrode and Swanson, 2004) using the full data in 2014.

\section{Single-Step Genomic Model}

As described in Aguilar et al. (2010), a mixed model equation (MME) for the ssGBLUP can be expressed as follows:

$$
\left[\begin{array}{cc}
\mathbf{X}^{\prime} \mathbf{X} & \mathbf{X}^{\prime} \mathbf{Z} \\
\mathbf{Z}^{\prime} \mathbf{X} & \mathbf{Z}^{\prime} \mathbf{Z}+\mathbf{H}^{-1} \alpha
\end{array}\right]\left[\begin{array}{l}
\mathbf{b} \\
\mathbf{a}
\end{array}\right]=\left[\begin{array}{c}
\mathbf{X}^{\prime} \mathbf{y} \\
\mathbf{Z}^{\prime} \mathbf{y}
\end{array}\right]
$$

In Equation [1], $\mathbf{y}$ is the vector for observations, $\mathbf{b}$ is the vector for fixed effects, $\mathbf{a}$ is the vector for random 
additive genetic effects, $\mathbf{X}$ is the design matrix for fixed effects, $\mathbf{Z}$ is the design matrix for random effects, $\alpha$ is the variance ratio $\left(\frac{\sigma_{e}^{2}}{\sigma_{a}^{2}}\right)$ of the residual variance $\sigma_{e}^{2}$ and the additive genetic variance $\sigma_{a}^{2}$, and $\mathbf{H}^{-1}$ is the inverse of the combined relationship matrix as below (Legarra et al., 2009; Aguilar et al., 2010):

$$
\mathbf{H}^{-1}=\left[\begin{array}{cc}
\mathbf{A}^{11} & \mathbf{A}^{12} \\
\left(\mathbf{A}^{12}\right)^{\prime} & \mathbf{A}^{22}
\end{array}\right]+\left[\begin{array}{cc}
0 & 0 \\
0 & \lambda\left(\tau \mathbf{G}^{-1}-\omega \mathbf{A}_{22}^{-1}\right)
\end{array}\right] .
$$

In Equation [2], $\mathbf{A}^{n n}$ is the inverse of the pedigree-based relationship matrix where $n=1$ stands for nongenotyped animals and $n=2$ stands for genotyped animals, $\mathbf{G}^{-1}$ is the inverse of the genomic relationship matrix, and $\lambda, \tau$, and $\omega$ are scaling factors on $\mathbf{G}^{-1}-\mathbf{A}_{22}^{-1}, \mathbf{G}^{-1}$, and $\mathbf{A}_{22}^{-1}$, which is the inverse of the pedigree-based relationship matrix for genotyped animals, respectively. Misztal et al. (2017) explained that these scaling parameters could indirectly account for missing pedigrees in $\mathbf{A}$.

In simulation scenarios (a) and (b), inbreeding coefficients were included in $\mathbf{A}$ for all animals and in $\mathbf{G}$ and $\mathbf{A}_{22}$ for genotyped animals. When $50 \%$ of dams were missing, approximated inbreeding coefficients including unknown dams were obtained for A. Likewise, in linear type traits, inbreeding coefficients were included in $\mathbf{A}$ as well as in $\mathbf{G}$ and $\mathbf{A}_{22}$. In this study, $\mathbf{G}$ as defined in VanRaden (2007) and VanRaden (2008) was used, which can have a similar scale as A. Although diagonals $<1.0$ and off-diagonals $<0.0$ in $\mathbf{G}$ were not present in $\mathbf{A}$, scales of these inverses were similar. Forni et al. (2011) showed high correlations (0.99) among GEBV using different definitions of $\mathbf{G}$, indicating that inverses of $\mathbf{G}$ were similar despite definitions of $\mathbf{G}$ being different.

\section{UPG for Combined Genomic Relationship Matrix H}

The main reason for assigning UPG in genetic evaluation is that the genetic trend could be significantly biased when ignoring genetic differences among genetic groups in a strongly selected population. Genetic groups can be defined based on genetic differences such as generations, sex, breeds, lines, families, and origins. Assigning UPG is one way to adjust the genetic difference. However, assigning UPG in genomic evaluation with a single-step algorithm is not straightforward. Using the QP transformation (Quaas and Pollak, 1981), genetic groups were assigned for unknown (phantom) parent(s) in Westell et al. (1988). As they described the QP transformation, the genetic group effect is the average contribution of unknown (phantom) parent(s) to their descendants that have phenotypic records (i.e., daughters in a sire model). In genomic predictions, descendants of some young genotyped sires and dams assigned in the UPG may not have daughters with phenotypes.

In Misztal et al. (2013), UPG was assigned in the genomic model as below:

Table 1. Numbers (percentage) of unknown parent groups and numbers of known parents in pedigree

\begin{tabular}{|c|c|c|c|c|}
\hline \multirow[b]{2}{*}{ Year of birth group } & \multicolumn{2}{|c|}{ No. of unknown parents (\%) } & \multicolumn{2}{|c|}{ No. of known parents } \\
\hline & Sire & Dam & Sire & Dam \\
\hline $\begin{array}{l}-1973 \\
\end{array}$ & $283,296(37)$ & $201,963(34)$ & 484,012 & 396,455 \\
\hline $1974-75$ & & $84,921(50)$ & & 83,969 \\
\hline $1976-77$ & $193,876(32)$ & $119,745(47)$ & 405,810 & 136,663 \\
\hline $1978-79$ & & $85,352(25)$ & & 257,926 \\
\hline $1980-81$ & $52.797(6)$ & $73.359(16)$ & 894.522 & 383.867 \\
\hline $1982-83$ & & $87,749(18)$ & & 402,344 \\
\hline $1984-85$ & $48,354(4)$ & $107,167(21)$ & $1,027,363$ & 407,242 \\
\hline $1986-87$ & & $135,050(24)$ & & 426,258 \\
\hline $1988-89$ & $38,289(3)$ & $143,349(25)$ & $1,108,489$ & 434,879 \\
\hline $1990-91$ & & $139,968(25)$ & & 428,582 \\
\hline $1992-93$ & $37,680(4)$ & $142,642(26)$ & $1,003,526$ & 401,985 \\
\hline $1994-95$ & & $140,439(28)$ & & 356,140 \\
\hline $1996-97$ & $63,728(7)$ & $153,791(32)$ & 890,750 & 332,665 \\
\hline 1998-99 & & $154,371(33)$ & & 313,651 \\
\hline $2000-01$ & $70,659(8)$ & $141,228(32)$ & 830,118 & 306,200 \\
\hline $2002-03$ & & $140,367(31)$ & & 312,982 \\
\hline $2004-05$ & $45,340(5)$ & $144,640(31)$ & 886,456 & 327,188 \\
\hline 2006-07 & & $127,327(28)$ & & 332,641 \\
\hline 2008-09 & $172.092(13)$ & $163.015(34)$ & $1,193.786$ & 317,727 \\
\hline $2010-$ & & $232,570(26)$ & & 652,566 \\
\hline Total & $1,006,111(10)$ & $2,719,013(28)$ & $8,724,832$ & $7,011,930$ \\
\hline
\end{tabular}




$$
\begin{aligned}
& \left.\begin{array}{rl}
\mathbf{Z}^{\prime} \mathbf{Z}+\left[\begin{array}{cc}
\mathbf{A}^{11} & \mathbf{A}^{12} \\
\left(\mathbf{A}^{12}\right)^{\prime} & \mathbf{A}^{22}
\end{array}\right]+\left[\begin{array}{cc}
0 & 0 \\
0 & \left(\mathbf{G}^{-1}-\mathbf{A}_{22}^{-1}\right)
\end{array}\right] & -\left(\left[\begin{array}{cc}
\mathbf{A}^{11} & \mathbf{A}^{12} \\
\left(\mathbf{A}^{12}\right)^{\prime} & \mathbf{A}^{22}
\end{array}\right]+\left[\begin{array}{cc}
0 & 0 \\
0 & \left(\mathbf{G}^{-1}-\mathbf{A}_{22}^{-1}\right)
\end{array}\right]\right) \mathbf{Q} \\
\text { sym } & \mathbf{Q}^{\prime}\left(\left[\begin{array}{cc}
\mathbf{A}^{11} & \mathbf{A}^{12} \\
\left(\mathbf{A}^{12}\right)^{\prime} & \mathbf{A}^{22}
\end{array}\right]+\left[\begin{array}{cc}
0 & 0 \\
0 & \left(\mathbf{G}^{-1}-\mathbf{A}_{22}^{-1}\right)
\end{array}\right]\right) \mathbf{Q}
\end{array}\right] \\
& =\left[\begin{array}{cc}
\mathbf{Z}^{\prime} \mathbf{Z}+\left[\begin{array}{cc}
\mathbf{A}^{11} & \mathbf{A}^{12} \\
\left(\mathbf{A}^{12}\right)^{\prime} & \mathbf{A}^{22}
\end{array}\right]+\left[\begin{array}{cc}
0 & 0 \\
0 & \left(\mathbf{G}^{-1}-\mathbf{A}_{22}^{-1}\right)
\end{array}\right] & -\left[\begin{array}{cc}
\mathbf{A}^{11} & \mathbf{A}^{12} \\
\left(\mathbf{A}^{12}\right)^{\prime} & \mathbf{A}^{22}
\end{array}\right] \mathbf{Q}_{1} \\
\text { sym } & \mathbf{Q}_{1}^{\prime}\left[\begin{array}{cc}
\mathbf{A}^{11} & \mathbf{A}^{12} \\
\left(\mathbf{A}^{12}\right)^{\prime} & \mathbf{A}^{22}
\end{array}\right] \mathbf{Q}_{1}
\end{array}\right] \\
& +\left[\begin{array}{cc}
0 & -\left[\begin{array}{cc}
0 & 0 \\
0 & \left(\mathbf{G}^{-1}-\mathbf{A}_{22}^{-1}\right)
\end{array}\right] \mathbf{Q}_{2} \\
\operatorname{sym} & \mathbf{Q}_{2}^{\prime}\left[\begin{array}{cc}
0 & 0 \\
0 & \left(\mathbf{G}^{-1}-\mathbf{A}_{22}^{-1}\right)
\end{array}\right] \mathbf{Q}_{2}
\end{array}\right]
\end{aligned}
$$$$
\left[\begin{array}{cc}
\mathbf{Z}^{\prime} \mathbf{Z}+\mathbf{H}^{-1} & -\mathbf{H}^{-1} \mathbf{Q} \\
-\mathbf{Q}^{\prime} \mathbf{H}^{-1} & \mathbf{Q}^{\prime} \mathbf{H}^{-1} \mathbf{Q}
\end{array}\right]\left[\begin{array}{c}
\mathbf{u} \\
\mathbf{g}
\end{array}\right]=\left[\begin{array}{c}
\mathbf{Z}^{\prime} \mathbf{y} \\
0
\end{array}\right],
$$

selected 15,000 genotyped animals as "core animals." [3] The APY is a method that calculates approximated $\mathbf{G}^{-1}$ for a large number of genotyped animals when the G matrix cannot be directly inverted. A small number of "core animals" are chosen to calculate $\mathbf{G}^{-1}$ directly.

where $\mathbf{Q}$ is the matrix that assigns UPG for genetic groups [i.e., birth-year groups and sex or generations for all missing parent(s) in this study], $\mathbf{u}$ is the vector of additive genetic effects, and $\mathbf{g}$ is the vector of UPG effects. The GEBV can be obtained by $\mathbf{Q g}+\mathbf{u}$. To solve Equation [3] for $\mathbf{g},-\mathbf{H}^{-1} \mathbf{Q}$ or $-\mathbf{Q}^{\prime} \mathbf{H}^{-1}$ must contain nonzero elements because the right-hand side corresponding to $\mathbf{g}$ is zero. If animals with and without phenotypes but sharing the same UPG are not related, $\mathbf{g}$ is not estimable. If animals in $\mathbf{A}^{22}$ are not related to animals in $\mathbf{A}^{11}$ (i.e., $\mathbf{A}^{12}=0$ ), the $\mathbf{H}^{-1}$ will not contribute to estimate g. As described by Misztal et al. (2013), the first part of the left-hand side in [3] can be split up into Equation [4], where $\mathbf{Q}_{1}$ and $\mathbf{Q}_{2}$ contain identifications linked to UPG for nongenotyped animals and for genotyped animals, respectively. In this study, after tuning $\mathbf{G}^{-1}$ to $\mathbf{A}_{22}^{-1}$ (i.e., matching means of diagonals and off-diagonals in $\mathbf{G}^{-1}$ to $\mathbf{A}_{22}^{-1}$ ), results of genomic predictions using $\mathbf{Q}_{2}^{\prime}\left(\mathbf{G}^{-1}-\mathbf{A}_{22}^{-1}\right) \mathbf{Q}_{2}=\mathbf{Q}_{2}^{\prime} \mathbf{G}^{-1} \mathbf{Q}_{2}-\mathbf{Q}_{2}^{\prime} \mathbf{A}_{22}^{-1} \mathbf{Q}_{2}$ and using only $-\mathbf{Q}_{2}^{\prime} \mathbf{A}_{22}^{-1} \mathbf{Q}_{2}$ in [4] were compared.

\section{Software}

Genomic predictions in simulation and in US Holsteins were calculated with the algorithm of proven and young (APY) by Misztal et al. (2014), using randomly Using the $\mathbf{G}^{-1}$ for "core animals" and genomic relationships of "core animals" with "noncore animals," which can be a large number of genotyped animals, the $\mathbf{G}^{-1}$ for "noncore animals" is approximated. The BLUP90IOD program (Tsuruta et al., 2001) was used to calculate (G)EBV with the APY. No genotyped animals were removed by quality controls such as low minor allele frequencies and parentage conflicts.

\section{RESULTS AND DISCUSSION}

\section{Simulation}

Inflation and Accuracy in GEBV. Tables 2 and 3 show validation results in the simulation study using $\mathrm{b}_{1}$ and $\mathrm{R}^{2}$ from TBV $=\mathrm{b}_{0}+\mathrm{b}_{1} \times(\mathrm{G}) \mathrm{EBV}$, respectively. With no missing pedigree information and after removing phenotypes from daughters in the last generation, $b_{1}$ on EBV for males in generations 8, 9, and 10 were close to 1.0 with and without BLUP selection, indicating no inflation. On the other hand, when males had no daughters and no siblings with phenotypes, $b_{1}$ $<0.9$ on GEBV for males represented inflation, especially in generation 10, implying that this inflation in GEBV was not due to missing pedigrees in $\mathbf{A}$. The difference between $b_{1}$ on GEBV with and without in- 
breeding in A (INB_A and No INB, respectively) was large for the selected population but small for the nonselected population, with standard deviations of $b_{1}$ ranging from \pm 0.01 to \pm 0.04 (Table 2). The inflation was magnified by BLUP selection. With $50 \%$ unknown dams, EBV were not predictable under selection. Males in generation 10 cannot have accurate parent averages (PA) instead of EBV when their parent(s) are unknown, whereas no inflation in GEBV were found when inbreeding for UPG was considered in $\mathbf{A}^{-1}$ (UPG_A). When ignoring inbreeding in $\mathbf{A}$ (No INB), large inflation was found in generation 10 , indicating that $\mathbf{A}^{22}$ and $\mathbf{A}_{22}^{-1}$ were not directly comparable without inbreeding. Males in generation 9, which had siblings but no daughters with phenotypes, had no inflation when considering inbreeding and ignoring UPG in $\mathbf{G}^{-1}$ (UPG_A). This result suggests that $\mathbf{Q}$ may not be completely separated into $\mathbf{Q}_{1}$ and $\mathbf{Q}_{2}$ in practice because $\mathbf{Q}$ is assigned for $\mathbf{A}$ (all animals in pedigree) but not for $\mathbf{G}$ and $\mathbf{A}_{22}$ (genotyped animals). With and without missing pedigree, $\mathrm{R}^{2}$ for GEBV were low in generation 10 because those males had no daughters (Table 3). Including UPG in $\mathbf{G}^{-1}$ (UPG_G) diminished the accuracy of GEBV. On the other hand, $\mathrm{R}^{2}$ for GEBV with no selection were higher, indicating that selection deteriorated accuracy in GEBV as well as inflation.

Bias in Genetic Trend. Estimates of UPG in simulation from the selected population are shown in Figure 2. The last UPG was not estimable for EBV because no phenotypes exist in the last generation. Likewise, the last UPG was not estimable for GEBV when including UPG for $\mathbf{G}^{-1}$ and $\mathbf{A}_{22}^{-1}$ (UPG_G) in addition to UPG for $\mathbf{A}^{-1}$. In contrast, the last $\mathrm{UPG}$ was estimated accurately for GEBV by excluding UPG for $\mathbf{G}^{-1}$ (UPG_A). Inaccurate UPG estimates could affect not only accuracy in $(\mathrm{G}) \mathrm{EBV}$ but also bias in genetic trend, which may be related to the intercept $b_{0}$ in the regression equation. After the adjustment with the genetic base, intercepts $b_{0}$ from all GEBV were small within 1 genetic standard deviation (results not shown).

Genetic trends for males were calculated for TBV and GEBV with UPG for $\mathbf{G}^{-1}$ and $\mathbf{A}_{22}^{-1}$ (UPG_G), with UPG for $\mathbf{A}_{22}^{-1}$ (UPG_A), and with no UPG, using the simulation scenario (a) under selection. In Figure 3, sires with progeny in generations 1 to 6 , which means that those sires were selected, were used in genetic trends. Drops in generations 7 to 10 in Figure 3 were due to using all genotyped males (with and without progeny) in generations 7 to 10, meaning that those sires were not selected. On the other hand, in Figure 4, only selected sires that had daughters were included in all generations; therefore, no drops were found in generations 7 to 9 , and no trends were shown in generation 10 when sires did not have daughters. The genetic trend in Figure 4 is the actual trend that can transmit genes to the next generation, whereas the genetic trend in Figure 3 is the false trend that cannot always transmit genes to the next generation. In this simulation, $90 \%$ of the genotyped males were not used for mating to produce offspring in the next generation. Hence, those $90 \%$ of the males were included in generations 7 to 10 in Figure 3, whereas the remaining $10 \%$ was included in generations 1 to 6 in Figure 3 and in all generations in Figure 4. If genotyped animals with no progeny or no phenotypes were included, the genetic trend would not reflect the phenotypic trend. Therefore, the genetic trend in Figure 3 could be misleading. When comparing genetic trends from EBV and GEBV, what kind of animals (e.g., males with or without progeny, females with or without phenotypes) are included in the genetic trend is essential. The genetic trend with no UPG was largely overpredicted in both figures. When including UPG in $\mathbf{G}^{-1}$ (UPG_G), the average GEBV dropped in the last generation, whereas the genetic trend excluding UPG in $\mathbf{G}^{-1}$ (UPG_A) was similar to that for TBV. Genetic trend for selected males were similar except for the overprediction of GEBV with no UPG. This simulation study showed that using inbreeding for UPG in A (UPG_A) was the best option. When no phenotypes are available for young genotyped animals, which always happens in dairy, UPG for $\mathbf{G}^{-1}$ (UPG_G) should not be included. When genetic groups are assigned for unknown parents in $\mathbf{A}, \mathbf{A}_{22}$ should have the same UPG. Plieschke et al. (2015) considered defining genetic groups in genomic BLUP using relationships based on SNP for multi-breed populations. When genetic differences among multi-breed populations are significant, including $\mathbf{A}_{22}^{-1}$ as well as $\mathbf{Q}_{2} \mathbf{G}^{-1} \mathbf{Q}_{2}$ may be useful to reduce bias in ssGBLUP.

\section{Linear Type Traits}

Inflation and Accuracy in GEBV. Regression coefficients $b_{1}$, which indicate inflation (overprediction in absolute values) of GEBV when $b_{1}<1.0$ or deflation (underprediction in absolute values) when $b_{1}>$ 1.0, were calculated with DYD in $2014=b_{0}+b_{1} \times$ GEBV in 2010 for 1,711 young genotyped bulls with no daughters in 2010 and at least 50 daughters in 2014 (Table 4). For all 18 type traits, $b_{1}$ for PA in 2010 was 0.76 on average, implying that PA was also inflated. One reason may be that PA for those young genotyped bulls was calculated by inflated GEBV of genotyped bulls with no daughters' phenotypes. 
TSURUTA ET AL.

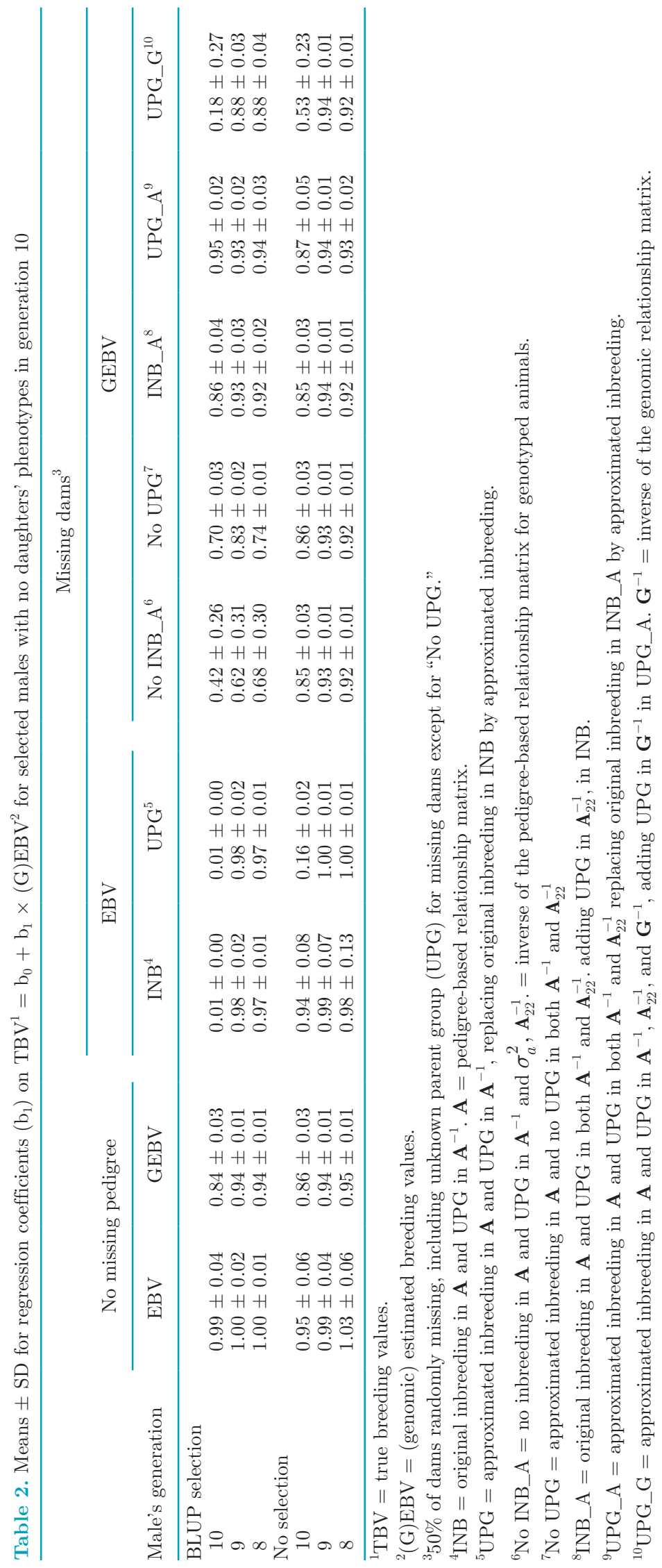




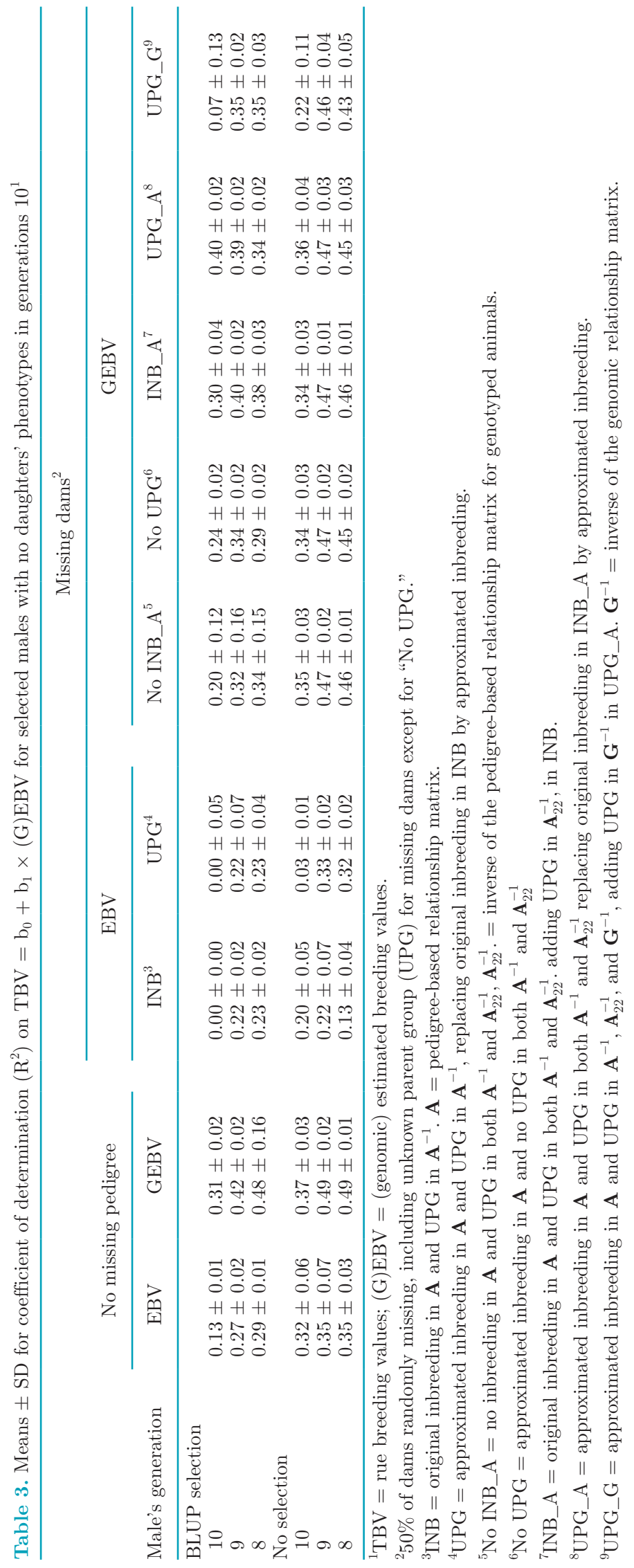




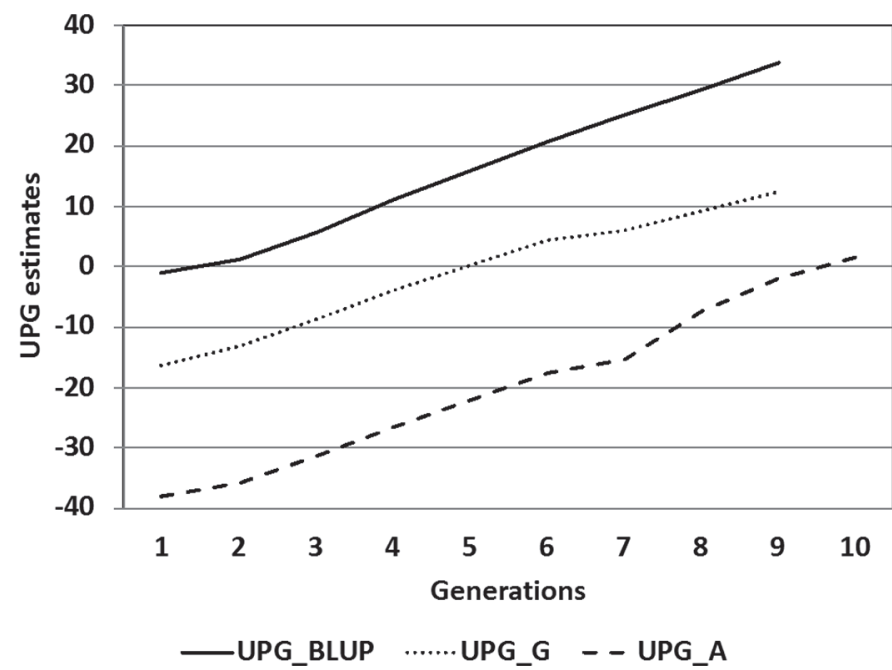

Figure 2. Unknown parent group (UPG) estimates in simulation. UPG_BLUP = without genotypes, UPG_G = including UPG for genomic relationships $(\mathbf{G})$ and pedigree-based relationships $\left(\mathbf{A}_{22}\right)$, and UPG_A = including UPG for $\mathbf{A}_{22}$ but not for $\mathbf{G}$; last UPG estimates for UPG_BLUP and UPG_G were omitted because they were not estimable in generation 10 .

The $b_{1}$ in GEBV was 0.85 on average when inbreeding was included in A (INB_A), increasing to 1.08 when approximated inbreeding for unknown parents was included in A (UPG_A). This result makes sense because the average inbreeding coefficient considering unknown parents (UPG_A) was higher than that not considering unknown parents (INB_A). Inflation in GEBV can be caused by a small $\mathbf{H}^{-1} \alpha$ in the left-hand

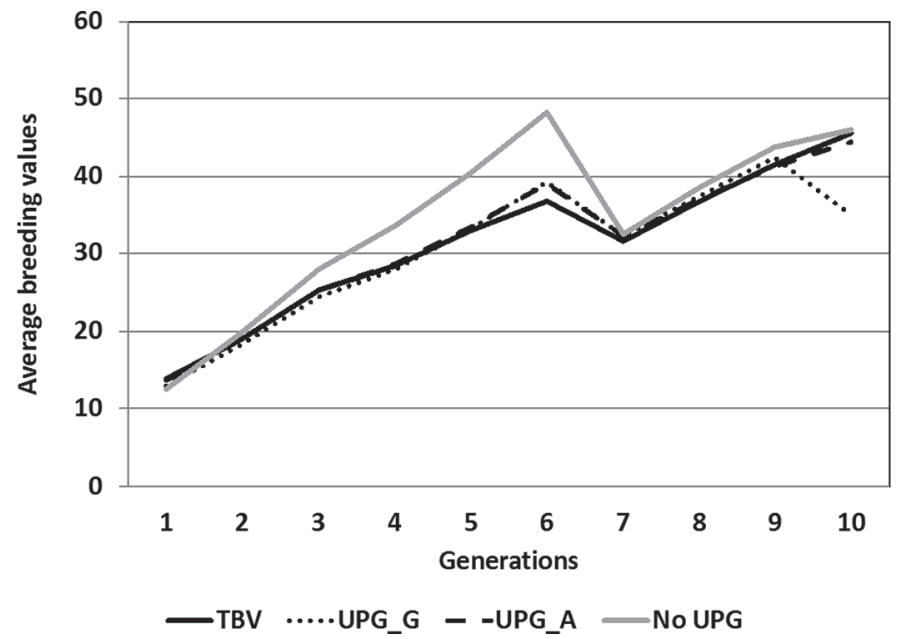

Figure 3. Genetic trend for sires with daughters in generations 1 to 6 and all genotyped males in generations 7 to 10 . TBV $=$ true breeding values; UPG_G = including unknown parent group (UPG) for genomic relationships $(\mathbf{G})$ and pedigree-based relationships $\left(\mathbf{A}_{22}\right)$; UPG_A = including UPG for $\mathbf{A}_{22}$ but not for $\mathbf{G}$; and No UPG $=$ not including UPG. side of [1] when solving the MME. The higher inbreeding makes $\mathbf{H}^{-1}$ larger. As a result, inflation in GEBV can be reduced. Current heritabilities that have been used in the national evaluation were estimated $15 \mathrm{yr}$ ago, and the most recent heritability estimates in 2014 were $2 \%$ lower on average for the 18 type traits, ranging from $2 \%$ increase to $6.5 \%$ decrease in percentage points (our unpublished data). Lower heritabilities or smaller additive genetic variances could result in less inflation in PA and GEBV because they increase $\alpha$. In addition, stronger selection that creates larger genetic gain on a trait may cause more inflation in GEBV as well as in PA. These results were supported by the simulation study as previously described. Selection may decrease additive genetic variances or heritabilities for some of the 18 type traits, but a further study will be needed to find out the reason why heritabilities declined. For validation, DYD in 2014 may not have provided realistic results because they were actual progeny yield deviations for sires but not like TBV in simulation, and $b_{1}$ were calculated using only 1,711 young genotyped bulls with a limited number of daughters. Furthermore, DYD were based on phenotypes from one generation later in 2014. Therefore, inflation in GEBV in 2010 may be partly due to the generation gap from the genetic progress.

Coefficients of determination on the regression analysis were also calculated to determine the accuracy of GEBV (Table 5). Compared with the average $\mathrm{R}^{2}$ for

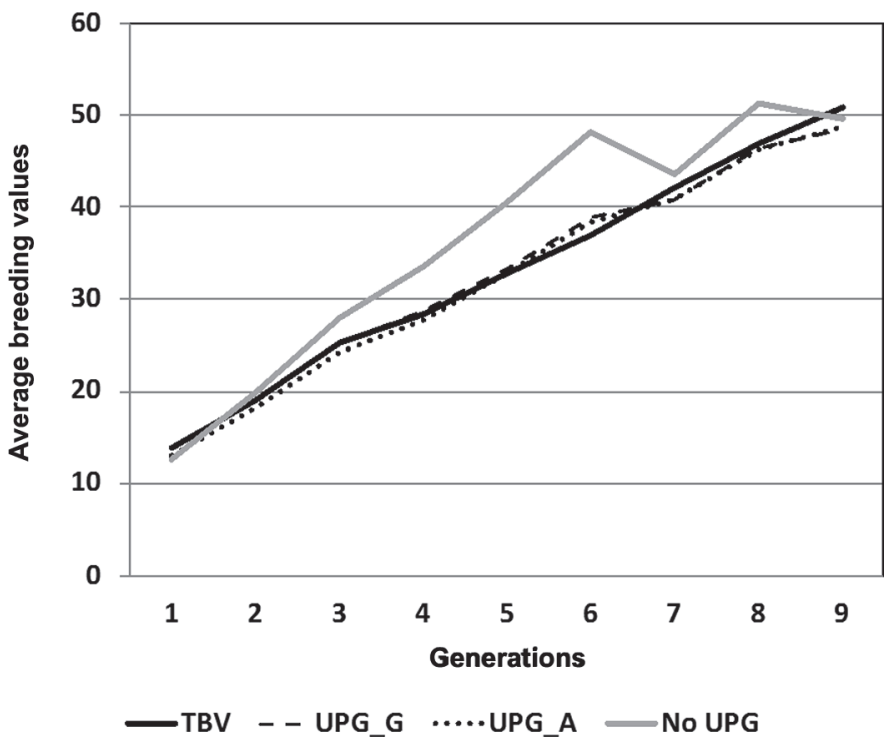

Figure 4. Genetic trend for sires with daughters in generations 1 to 6 and genotyped sires with daughters in generations 7 to 10 . TBV = true breeding values; UPG_G = including unknown parent group (UPG) for genomic relationships $(\mathbf{G})$ and pedigree-based relationships $\left(\mathbf{A}_{22}\right)$; UPG_A = including UPG for $\mathbf{A}_{22}$ but not for $\mathbf{G}$, and No UPG $=$ not including UPG. 
PA, GEBV were $31 \%$ and $26 \%$ more accurate than PA for young genotyped bulls with no daughters in 2010, when including original inbreeding coefficients and approximated inbreeding coefficients for unknown parents, respectively. The average $\mathrm{R}^{2}$ for GEBV with inbreeding in A (INB_A) provided the highest value (50\%). Because inbreeding coefficients in $\mathbf{A}$ were lower than those with UPG in $\mathbf{A}$ (UPG_A) on average, $\mathbf{H}^{-1} \alpha$ became smaller, which was equivalent to a smaller $\alpha$ and higher heritability. As a result, GEBV for INB_A could have a higher accuracy than GEBV for UPG_A in Table 5 as well as more inflation in Table 4. Including UPG in $\mathbf{G}^{-1}$ (UPG_G) did not show any different results than those from UPG in $\mathbf{A}^{-1}$ and $\mathbf{Q}_{2}^{\prime} \mathbf{A}_{22}^{-1} \mathbf{Q}_{2}$ (UPG_A) contrary to the results from the simulation, suggesting that inaccurate UPG estimates do not affect inflation and accuracy based on $\mathrm{b}_{1}$ and $\mathrm{R}^{2}$ in GEBV for type traits.

Bias in Genetic Trend. In the national evaluation model for type traits in US Holsteins, UPG were defined as in Table 1. To estimate UPG accurately, each group should have a sufficient number of animals (e.g., $>100$ ) with phenotypes. For type traits in US Holsteins, sires for all registered animals are known, but some dams may be unknown. Also, ancestors of sires may be unknown, especially if they are from foreign pedigrees. Young genotyped animals including heifers with temporary identifications may have unknown parents. Using the original definition of UPG (original), UPG estimates with EBV were not estimable in the last group for both dams and sires in 2010 (Figures 5 and 6). As reported by Tsuruta et al. (2014), the last UPG was not estimable and dropped because of no phenotypes in the last group, and by combining the last 2 groups, those drops disappeared (combined). However, without combining the last groups and by ignoring UPG for $\mathbf{G}^{-1}$, the drops with GEBV in the last groups of sires and dams in 2010 disappeared (No UPG for G). In genomic predictions, UPG became estimable without phenotypes. The UPG definition is based on the structure of pedigree-based relationships $\mathbf{A}$, whereas most of the elements in $\mathbf{G}$ are nonzero, implying no unknown

Table 4. Regression coefficient $\left(\mathrm{b}_{1}\right)$ on $\mathrm{DYD}^{1}=\mathrm{b}_{0}+\mathrm{b}_{1} \times \mathrm{GEBV}^{2}$ for young genotyped bulls with no daughters in 2010 and at least 50 daughters in 2014

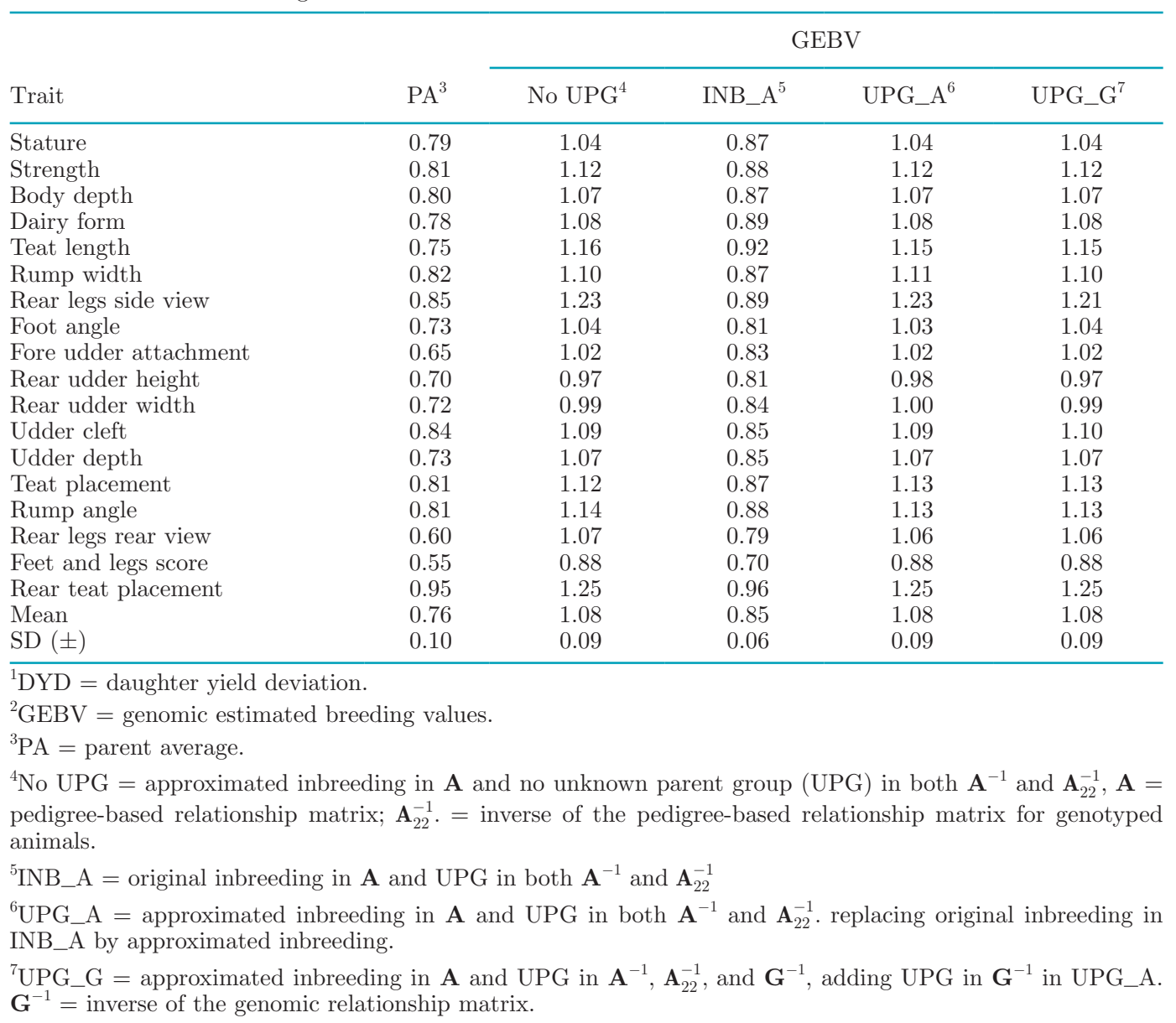


Table 5. Coefficient of determination on DYD $=b_{0}+b_{1} \times$ GEBV for young genotyped bulls with no daughters in 2010 and at least 50 daughters in $2014^{1}$

\begin{tabular}{|c|c|c|c|c|c|}
\hline \multirow[b]{2}{*}{ Trait } & \multirow[b]{2}{*}{$\mathrm{PA}^{2}$} & \multicolumn{4}{|c|}{ GEBV } \\
\hline & & No $U_{P G}^{3}$ & INB_A $A^{4}$ & UPG_A ${ }^{5}$ & UPG_G ${ }^{6}$ \\
\hline Stature & 0.32 & 0.63 & 0.67 & 0.64 & 0.64 \\
\hline Strength & 0.21 & 0.48 & 0.51 & 0.48 & 0.48 \\
\hline Body depth & 0.28 & 0.57 & 0.61 & 0.58 & 0.58 \\
\hline Dairy form & 0.24 & 0.53 & 0.60 & 0.54 & 0.54 \\
\hline Teat length & 0.19 & 0.52 & 0.59 & 0.52 & 0.52 \\
\hline Rump width & 0.22 & 0.47 & 0.53 & 0.48 & 0.48 \\
\hline Rear legs side view & 0.18 & 0.37 & 0.40 & 0.37 & 0.37 \\
\hline Foot angle & 0.15 & 0.29 & 0.35 & 0.29 & 0.29 \\
\hline Fore udder attachment & 0.17 & 0.46 & 0.52 & 0.47 & 0.47 \\
\hline Rear udder height & 0.20 & 0.41 & 0.49 & 0.43 & 0.43 \\
\hline Rear udder width & 0.17 & 0.35 & 0.46 & 0.38 & 0.38 \\
\hline Udder cleft & 0.20 & 0.40 & 0.45 & 0.40 & 0.40 \\
\hline Udder depth & 0.17 & 0.49 & 0.53 & 0.50 & 0.50 \\
\hline Teat placement & 0.18 & 0.46 & 0.51 & 0.46 & 0.46 \\
\hline Rump angle & 0.22 & 0.46 & 0.54 & 0.47 & 0.47 \\
\hline Rear legs rear view & 0.07 & 0.22 & 0.27 & 0.22 & 0.22 \\
\hline Feet and legs score & 0.14 & 0.35 & 0.41 & 0.37 & 0.37 \\
\hline Rear teat placement & 0.18 & 0.41 & 0.48 & 0.41 & 0.41 \\
\hline Mean & 0.19 & 0.44 & 0.50 & 0.45 & 0.45 \\
\hline $\mathrm{SD}( \pm)$ & 0.05 & 0.10 & 0.10 & 0.10 & 0.10 \\
\hline \multicolumn{6}{|c|}{$\begin{array}{l}{ }^{1} \mathrm{DYD}=\text { daughter yield deviation; } \mathbf{b}_{1}=\text { regression coefficient; GEBV }=\text { genomic estimated breeding values. } \\
{ }^{2} \mathrm{PA}=\text { parent average. }\end{array}$} \\
\hline \multicolumn{6}{|c|}{$\begin{array}{l}{ }^{3} \text { No } U P G=\text { approximated inbreeding in } \mathbf{A} \text { and no unknown parent group (UPG) in both } \mathbf{A}^{-1} \text { and } \mathbf{A}_{22}^{-1}, \mathbf{A}= \\
\text { pedigree-based relationship matrix; } \mathbf{A}_{22}^{-1} \text {. = inverse of the pedigree-based relationship matrix for genotyped } \\
\text { animals. }\end{array}$} \\
\hline \multicolumn{6}{|c|}{${ }^{4} \mathrm{INB} \_\mathrm{A}=$ original inbreeding in $\mathbf{A}$ and $\mathrm{UPG}$ in both $\mathbf{A}^{-1}$ and $\mathbf{A}_{22}^{-1}$} \\
\hline \multicolumn{6}{|c|}{$\begin{array}{l}{ }^{5} \mathrm{UPG} \text { _A }=\text { approximated inbreeding in } \mathbf{A} \text { and } \mathrm{UPG} \text { in both } \mathbf{A}^{-1} \text { and } \mathbf{A}_{22}^{-1} \text {. replacing original inbreeding in } \\
\text { INB_A by approximated inbreeding. }\end{array}$} \\
\hline
\end{tabular}

pedigree information in $\mathbf{G}$. The same UPG definition in A may not apply in $\mathbf{G}$. Therefore, including UPG for $\mathbf{G}^{-1}$, which is $\mathbf{A}_{22}^{-1}$ in [4], could hinder the ability to estimate UPG accurately. The inestimable UPG in $\mathbf{A}$ and $\mathbf{A}_{22}$ could be cancelled out. When genotypes from multiple base populations such as different breeds or species are used to construct $\mathbf{G}$, considering genetic groups in $\mathbf{G}$ could be useful to account for genetic differences.

Figures 7 and 8 show genetic trends for stature and udder depth as examples from 18 traits, respectively, in genotyped bulls with at least 50 daughters in 2014, assigning UPG for $\mathbf{G}$ and $\mathbf{A}_{22}$ (UPG_G10 and UPG_G14) or assigning UPG for $\mathbf{A}_{22}$ (UPG_A10 and UPG_A14), in addition to the original UPG in $\mathbf{A}$ defined in Table 1. All genetic trends were adjusted with the genetic base, which were calculated based on $(\mathrm{G}) \mathrm{EBV}$ of cows born in 2005. The genetic trend for stature using UPG for $\mathbf{G}^{-1}$ and $\mathbf{Q}_{2} \mathbf{G}^{-1} \mathbf{Q}_{2}$ in 2010 data (UPG_G10) was underestimated in the last $4 \mathrm{yr}$ when most of the young genotyped bulls were born. Similarly, the genetic trend for udder depth using UPG for $\mathbf{G}^{-1}$ and $\mathbf{A}_{22}^{-1}$ in 2010 data (UPG_G10) was underestimated. These underestimates could be attributed to inaccurate UPG estimates. Slight underestimation of the genetic trend for udder depth using UPG for $\mathbf{A}_{22}^{-1}$ in 2010 data (UPG_ A10) could be due to strong selection on udder depth.

\section{Other Approaches to Controlling Bias in GEBV}

In the preliminary analysis of this study, the program did not converge for 18 type traits when ignoring inbreeding in $\mathbf{A}$ even though the smallest eigenvalue of the additive genetic covariance matrix for 18 traits explained $0.05 \%$ of the variance, and the matrix was positive definite. However, when solving the MME with multiple trait models, the left-hand side of the MME (1) can be nearly ill-conditioned when the covariance matrix is close to nonpositive definite and $\mathbf{A}^{22}, \mathbf{G}^{-1}$, and $\mathbf{A}_{22}^{-1}$ are incompatible, creating a smaller $\mathbf{H}^{-1} \alpha . \mathbf{A}$ smaller $\omega<1.0$ for $\mathbf{A}_{22}^{-1}$ which increases $\mathbf{H}^{-1}$, helps the program converge. Using other scaling factors $\lambda$ and $\tau$ 

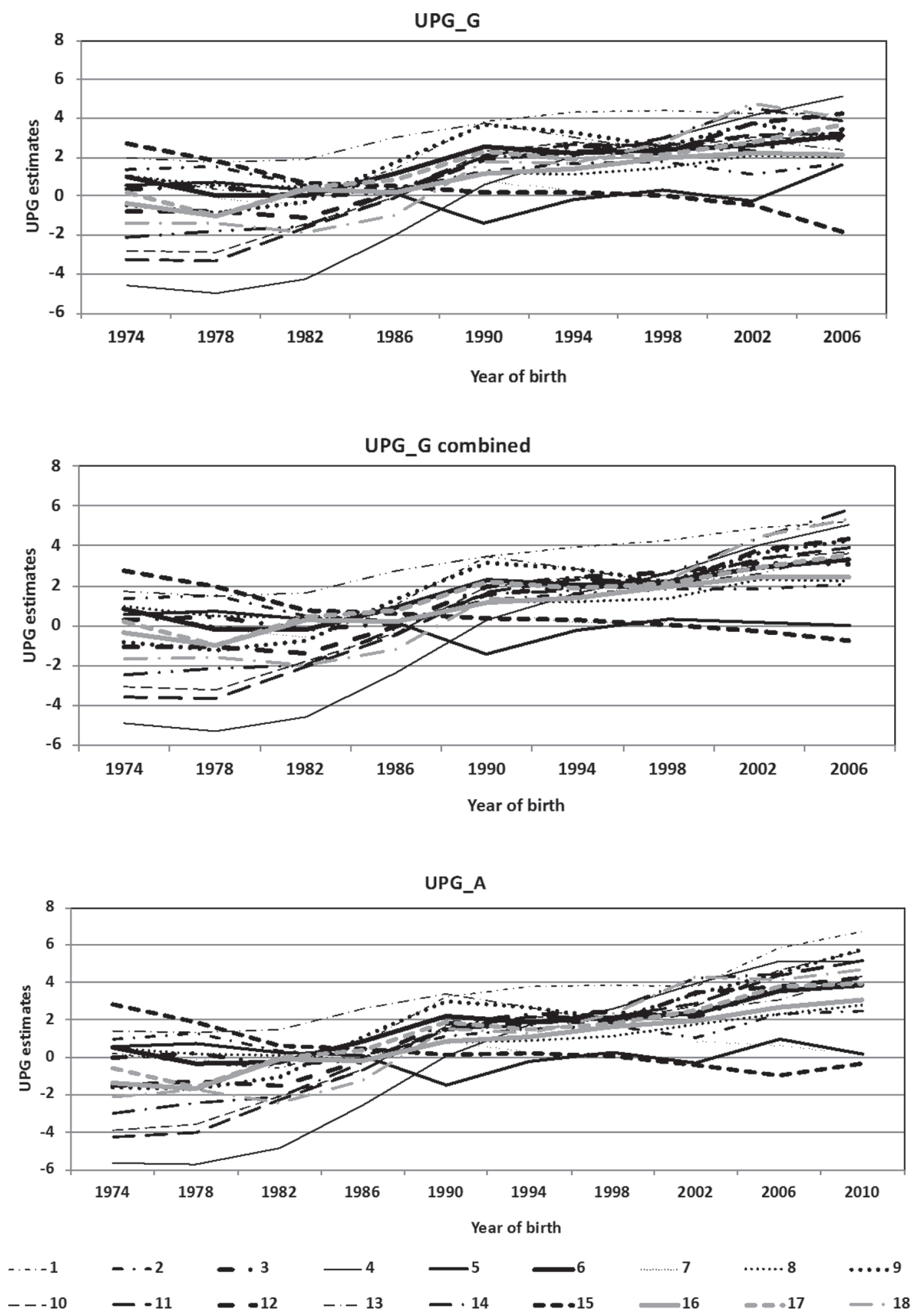

Figure 5. Unknown parent group (UPG) estimates for sires: original definition in $\mathbf{A}$ with genomic relationships $(\mathbf{G})$ and pedigree-based relationships ( $\mathbf{A}_{22}$; UPG_G), UPG_G with combined last 2 groups (UPG_G combined), and original definition in $\mathbf{A}$ with $\mathbf{A}_{22}$ but no $\mathbf{G}$ (UPG_A). Traits: $1=$ stature, $2=$ strength, $3=$ body depth, $4=$ dairy form, $5=$ teat length, $6=$ rump width, $7=$ rear legs side view, $8=$ foot angle, 9 $=$ fore udder attachment, $10=$ rear udder height, $11=$ rear udder width, $12=$ udder cleft, $13=$ udder depth, $14=$ teat placement, $15=$ rump angle, $16=$ rear legs rear view, $17=$ feet and legs score, $18=$ rear teat placement. 


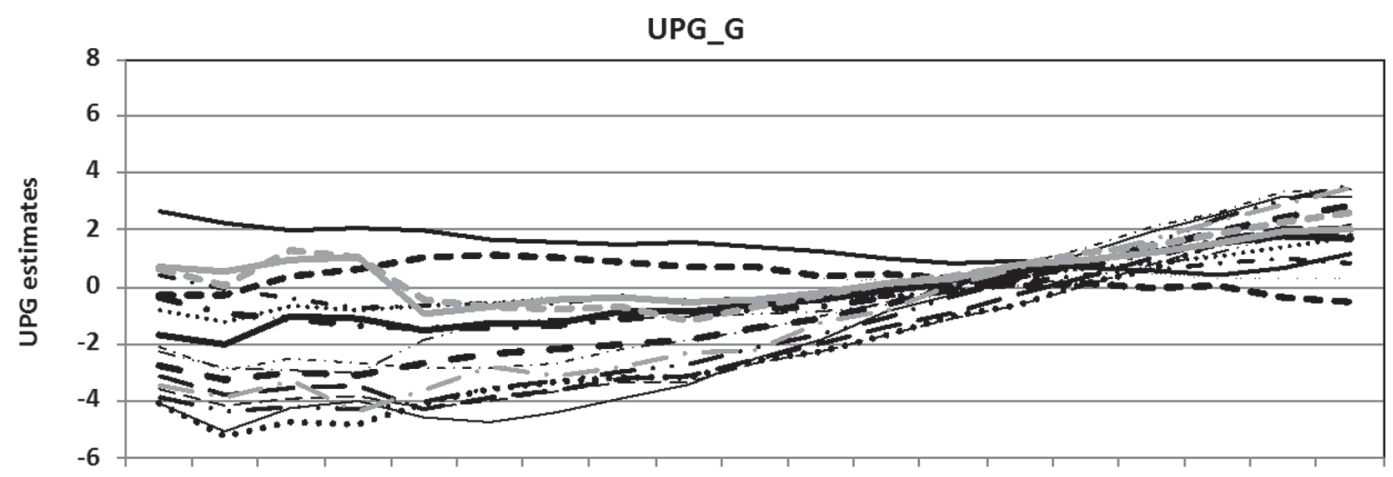

1972197419761978198019821984198619881990199219941996199820002002200420062008

Year of birth

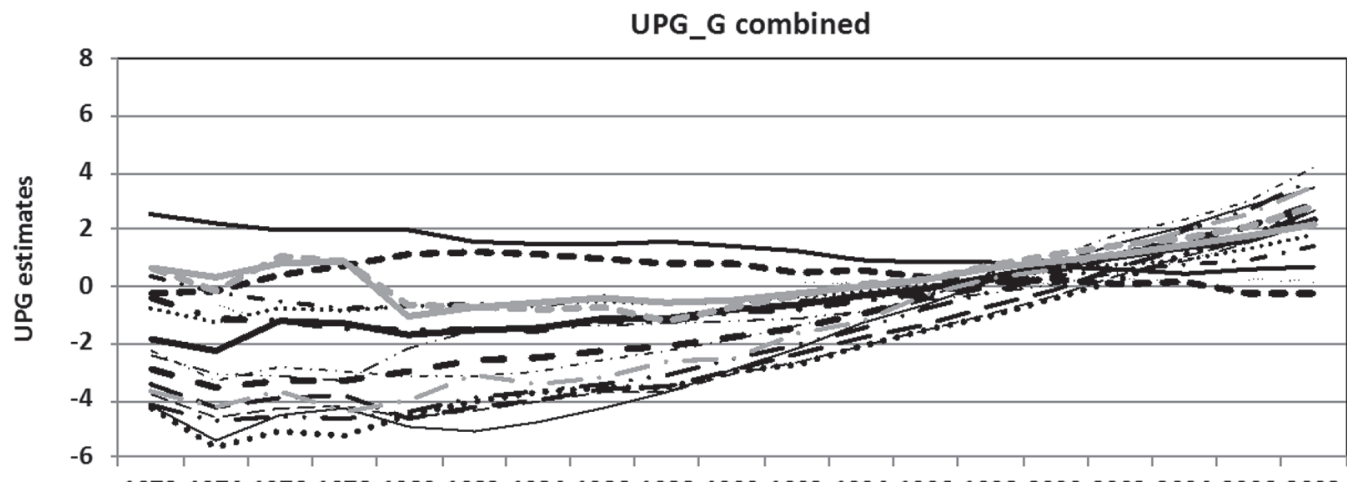

1972197419761978198019821984198619881990199219941996199820002002200420062008

Year of birth

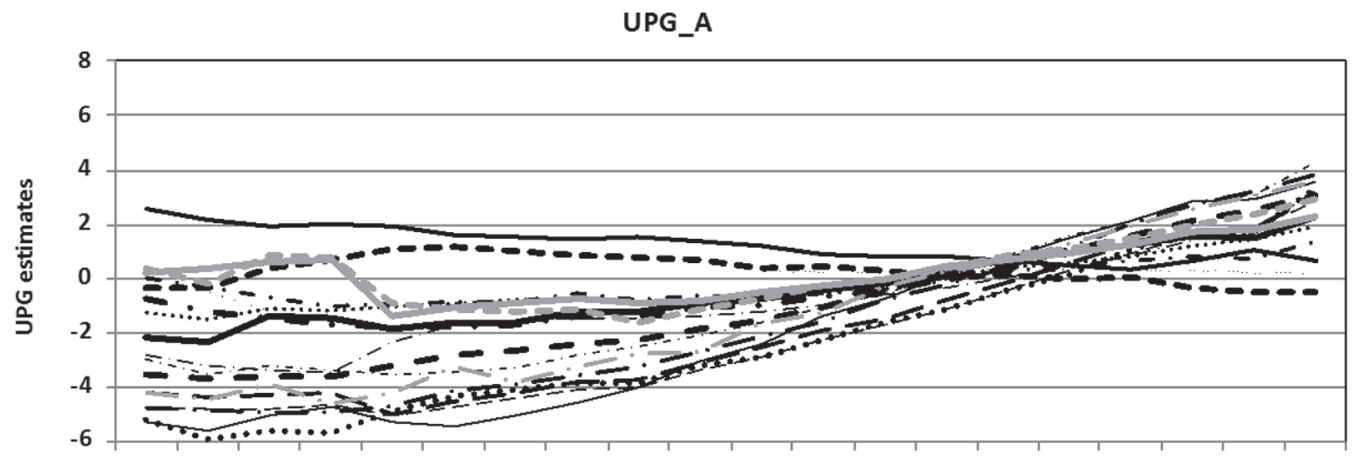

19721974197619781980198219841986198819901992199419961998200020022004200620082010

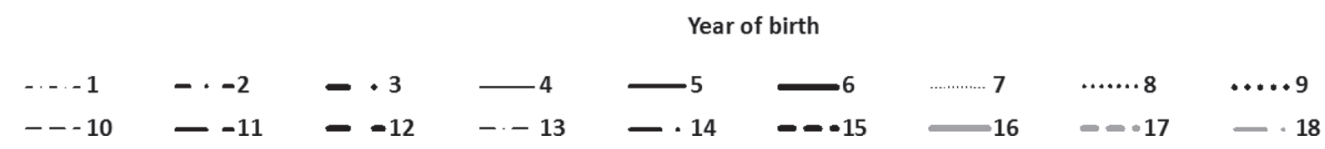

Figure 6. Unknown parent group (UPG) estimates for dams: original definition in $\mathbf{A}$ with genomic relationships $(\mathbf{G})$ and pedigree-based relationships ( $\mathbf{A}_{22} ; \mathrm{UPG}$ _G), UPG_G with combined last 2 groups (UPG_G combined), and original definition in $\mathbf{A}$ with $\mathbf{A}_{22}$ but no $\mathbf{G}$ (UPG_A). Traits: $1=$ stature, $2=$ strength, $3=$ body depth, $4=$ dairy form, $5=$ teat length, $6=$ rump width, $7=$ rear legs side view, $8=$ foot angle, 9 = fore udder attachment, $10=$ rear udder height, $11=$ rear udder width, $12=$ udder cleft, $13=$ udder depth, $14=$ teat placement, $15=$ rump angle, $16=$ rear legs rear view, $17=$ feet and legs score, $18=$ rear teat placement.

in [2] for $\mathbf{A}_{22}^{-1}$, and $\mathbf{G}^{-1}$, respectively, could also reduce the inflation, by increasing $\mathbf{H}^{-1}$. For the 18 type traits, when ignoring $\left(\mathbf{G}^{-1}-\mathbf{A}_{22}^{-1}\right)$ a scaling factor $\omega=0.9$ on
$\mathbf{Q}_{2}^{\prime}\left(\mathbf{G}^{-1}-\mathbf{A}_{22}^{-1}\right) \mathbf{Q}_{2}$, created better compatibility among $\mathbf{A}^{22}, \mathbf{G}^{-1}$, and $\mathbf{A}_{22}^{-1}$ to predict GEBV, which is similar to using higher inbreeding coefficients. The $b_{1}$ can increase 
to 1.02 without inbreeding in $\mathbf{A}$ when using $\omega<1.0$ on $\mathbf{A}_{22}^{-1}$ (these results are not included in Tables 4 and 5 ). Controlling the incompatibility by changing scaling factors is one way to make convergence possible and fast as well as reduce inflation in GEBV (e.g., using $\omega<$ 1.0). Incompatibility among inverses of these relationship matrices after the base adjustment can be corrected with the scaling factors, but different scaling factors may be required in each trait, each model, and each population. It will be difficult to obtain the most appropriate scaling factor in every circumstance.

Different bases of $\mathbf{A}$ or $\mathbf{A}_{22}$ and $\mathbf{G}$ can be adjusted by tuning, but distributions of $\mathbf{A}$ or $\mathbf{A}_{22}$ and $\mathbf{G}$ are not comparable due to different definitions of these relationship matrices. Christensen (2012) proposed the adjustment of $\mathbf{G}$ to $\mathbf{A}$ by reconstructing $\mathbf{H}$ but not $\mathbf{H}^{-1}$. Using metafounders could be the method of choice to improve the compatibility between $\mathbf{A}$ and $\mathbf{G}$ in the single-step genomic algorithm (Legarra et al., 2015) if it is computationally practical.

Increasing $\alpha=\mathbf{A}_{22}^{-1}$ is another way to reduce the inflation; that is, increasing $\frac{\sigma_{e}^{2}}{\sigma_{a}^{2}}$ decreasing $\sigma_{e}^{2}$, or decreasing the heritability. Wiggans et al. (2012) showed that decreasing the heritability by $50 \%$ decreases the bias. Using smaller additive genetic variances or lower heritabilities can reduce inflation in GEBV (Tsuruta et al., 2018), but it may not be acceptable because GEBV and

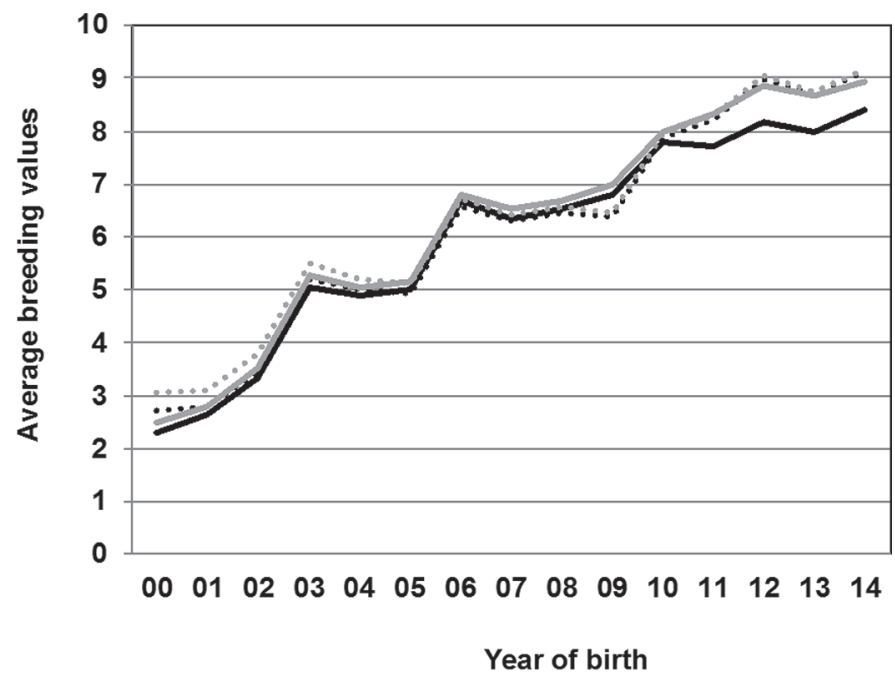

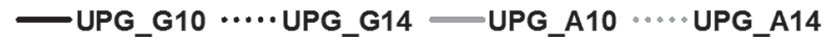

Figure 7. Genetic trend for genotyped bulls with at least 50 daughters in 2014 for stature: UPG_G10 and UPG_G14 including unknown parent group (UPG) for genomic relationships $(\mathbf{G})$ and pedigree-based relationships $\left(\mathbf{A}_{22}\right)$ in 2010 and 2014 data, respectively; UPG_A10 and UPG_A14 using UPG for pedigree-based relationships $\left(\mathbf{A}_{22}\right)$ in 2010 and 2014 data, respectively.

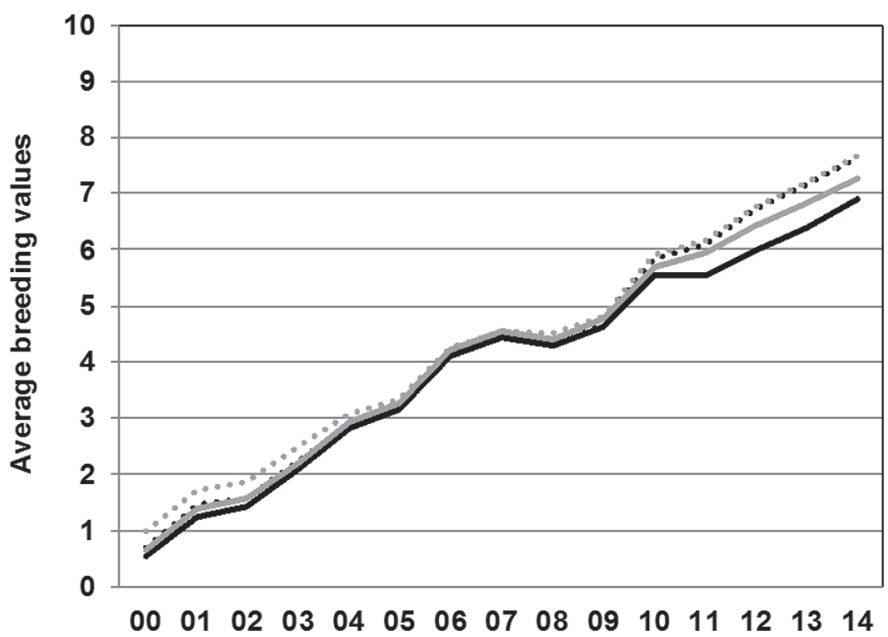

Year of birth

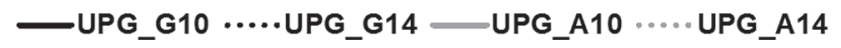

Figure 8. Genetic trend for genotyped bulls with at least 50 daughters in 2014 for udder depth: UPG_G10 and UPG_G14 including unknown parent group (UPG) for genomic relationships $(\mathbf{G})$ and pedigree-based relationships $\left(\mathbf{A}_{22}\right)$ in 2010 and 2014 data, respectively; UPG_A10 and UPG_A14 using UPG for pedigree-based relationships $\left(\mathbf{A}_{22}\right)$ in 2010 and 2014 data, respectively.

reliabilities for all animals will shrink, resulting in smaller genetic trend, unless changes in (co)variance components over generations are considered in the model. When heritability and genetic correlations change over time, the additive genetic covariance should reflect the genetic structure of the current population. One approach to consider these parameter changes over time is to use a random-regression model or a multiple trait model on years (Tsuruta et al., 2002), but it will be computationally heavy in a large population. Genetic parameters should be periodically renewed, and at least genomic predictions for young animals as well as all active animals should be calculated using the up-to-date genetic parameters.

\section{CONCLUSIONS}

It is crucial to ensure compatibility between pedigreebased and genomic relationships for unbiased genomic predictions using a single-step genomic evaluation method. Ignoring inbreeding could be responsible for a large part of inflation in genomic predictions, especially when inbreeding is high in a selected population. Strong selection on a trait amplifies inflation in genomic predictions, especially when the incompatibility is large. Using approximated inbreeding coefficients considering unknown parents, inflation in genomic predictions can be further reduced. If UPG are not defined well 
(i.e., a small number of animals in an UPG or assigning UPG in the genomic relationship matrix for young genotyped animals with no progeny or no phenotypes), they will not be estimated accurately or will not be estimable. Inaccurate estimates of UPG can create bias in genomic predictions and genetic trends, although inflation in genomic predictions in type traits for Holsteins was not evident. Taking into account the genetic parameters changing over time or across populations in genomic predictions will be a future research topic. It is possible to reduce inflation in genomic predictions for young genotyped animals by using smaller additive genetic variances, adjusting the weight of the relationship matrix for genotyped animals, or both. Additive genetic variances can be shrunk under selection; therefore, recently estimated genetic parameters should be used to predict genomic breeding values. Without manipulating additive genetic variances, bias in genomic prediction can be reduced by including inbreeding in the pedigree-based relationship matrix and using latest genetic parameters. The results in this study may be applicable to genomic predictions in other breeds and species.

\section{ACKNOWLEDGMENTS}

This study was partially funded by the Holstein Association USA Inc. (Brattleboro, VT) The authors thank USDA-Agricultural Research Service for providing genomic data.

\section{REFERENCES}

Aguilar, I., and I. Misztal. 2008. Recursive algorithm for inbreeding coefficients assuming non-zero inbreeding of unknown parents. J. Dairy Sci. 91:1669-1672.

Aguilar, I., I. Misztal, D. L. Johnson, A. Legarra, S. Tsuruta, and T. J. Lawlor. 2010. Hot topic: A unified approach to utilize phenotypic, full pedigree and genomic information for genetic evaluation of Holstein final score. J. Dairy Sci. 93:743-752.

Christensen, O. F. 2012. Compatibility of pedigree-based and marker-based relationship matrices for single-step genetic evaluation. Genet. Sel. Evol. 44:37.

Forni, S., I. Aguilar, and I. Misztal. 2011. Different genomic relationship matrices for single-step analysis using phenotypic, pedigree and genomic information. Genet. Sel. Evol. 43:1.

Hayes, B., and M. E. Goddard. 2001. The distribution of the effects of genes affecting quantitative traits in livestock. Genet. Sel. Evol. 33:209-229.

Koivula, M., I. Strandén, G. P. Aamand, and E. A. Mäntysaari. 2018. Reducing bias in the dairy cattle single-step genomic evaluation by ignoring bulls without progeny. J. Anim. Breed. Genet. 135:107115 .
Legarra, A., I. Aguilar, and I. Misztal. 2009. A relationship matrix including full pedigree and genomic information. J. Dairy Sci. 92:4656-4663.

Legarra, A., O. F. Christensen, Z. G. Vitezica, I. Aguilar, and I. Misztal. 2015. Ancestral relationships using metafounders: Finite ancestral populations and across population relationships. Genetics 200:455-468.

Masuda, Y., P. M. VanRaden, I. Misztal, and T. J. Lawlor. 2018. Differing genetic trend estimates from traditional and genomic evaluations of genotyped animals as evidence of preselection bias in US Holsteins. J. Dairy Sci. 101:5194-5206.

Misztal, I., Z. G. Vitezica, A. Legarra, I. Aguilar, and A. A. Swan. 2013. Unknown-parent groups in single-step genomic evaluation. J. Anim. Breed. Genet. 130:252-258.

Misztal, I., H. L. Bradford, D. A. L. Lourenco, S. Tsuruta, Y. Masuda, A. Legarra, and T. J. Lawlor. 2017. Studies on inflation of GEBV in single-step GBLUP for type. Interbull Bull. 51:38-42. Interbull meeting. Tallinn, Estonia.

Misztal, I., A. Legarra, and I. Aguilar. 2014. Using recursion to compute the inverse of the genomic relationship matrix. J. Dairy Sci 97:3943-3952

Mrode, R. A., and G. J. T. Swanson. 2004. Calculating cow and daughter yield deviations and partitioning of genetic evaluations under a random regression model. Livest. Prod. Sci. 86:253-260.

Plieschke, L., C. Edel, E. C. G. Pimentel, R. Emmerling, J. Bennewitz, and K. Götz. 2015. A simple method to separate base population and segregation effects in genomic relationship matrices. Genet. Sel. Evol. 47:53

Quaas, R. L., and E. J. Pollak. 1981. Modified equations for sire models with groups. J. Dairy Sci. 64:1868-1872.

Sargolzaei, M., and F. S. Schenkel. 2009. QMSim: A large-scale genome simulator for livestock. Bioinformatics 25:680-681.

Tsuruta, S., D. Lourenco, I. Misztal, and T. Lawlor. 2018. Possible causes of inflation in genomic evaluations for dairy cattle. In Proc. 11th World Congress on Genetics Applied to Livestock Production, Auckland, New Zealand. Accessed Aug. 22, 2019. http:// www.wcgalp.org/system/files/proceedings/2018/possible-causes -inflation-genomic-evaluations-dairy-cattle.pdf.

Tsuruta, S., I. Misztal, I. Aguilar, and T. J. Lawlor. 2011. Multipletrait genomic evaluation of linear type traits using genomic and phenotypic data in Holsteins. J. Dairy Sci. 94:4198-4204.

Tsuruta, S., I. Misztal, L. Klei, and T. J. Lawlor. 2002. Analysis of age-specific predicted transmitting abilities for final scores in Holsteins with a random regression model. J. Dairy Sci. 85:1324-1330.

Tsuruta, S., I. Misztal, D. A. L. Lourenco, and T. J. Lawlor. 2014 Assigning unknown parent groups to reduce bias in genomic evaluations of final score in US Holsteins. J. Dairy Sci. 97:5814-5821.

Tsuruta, S., I. Misztal, and I. Strandén. 2001. Use of the preconditioned conjugate gradient algorithm as a generic solver for mixed model-equations in animal breeding applications. J. Anim. Sci 79:1166-1172.

VanRaden, P. M. 2007. Genomic measures of relationship and inbreeding. Interbull Bull. 37:33-36. Interbull meeting. Dublin, Ireland

VanRaden, P. M. 2008. Efficient methods to compute genomic predictions. J. Dairy Sci. 91:4414-4423.

Vitezica, Z. G., I. Aguilar, I. Misztal, and A. Legarra. 2011. Bias in genomic predictions for populations under selection. Genet. Res. (Camb.) 93:357-366.

Westell, R. A., R. L. Quaas, and L. D. Van Vleck. 1988. Genetic groups in an animal model. J. Dairy Sci. 71:1310-1318.

Wiggans, G. R., P. M. VanRaden, and T. A. Cooper. 2012. Technical note: Adjustment of all cow evaluations for yield traits to be comparable with bull evaluations. J. Dairy Sci. 95:3444-3447. 